\title{
ANÁLISIS DE EFICIENCIA POR PROGRAMAS EN EL SECTOR DE LA ECONOMÍA SOCIAL: EL CASO DEL PRINCIPADO DE ASTURIAS
}

\author{
POR \\ Isidoro GUZMÁN RAJA ${ }^{1}$, \\ Aydee HURTADO GARCÉS ${ }^{2}$ y \\ Carmen RAMOS CARVAJAL ${ }^{3}$
}

\section{RESUMEN}

El presente trabajo evalúa la eficiencia del Sector de la Economía Social (SES) en Asturias, para lo cual se seleccionó el método no paramétrico del Análisis Envolvente de Datos (Data Envelopment Analysis, DEA) propuesto por Charnes et al. (1978). A partir de los datos extraídos de la cuentas anuales de una muestra de 397 empresas para el bienio 2004-2005, compuesta por sociedades cooperativas y laborales dada su relevancia en el SES asturiano, se aplicó la técnica DEA a un modelo de rendimiento basado en la cuenta de resultados de explotación bajo orientación output, utilizando para ello la metodología por programas propuesta por Charnes et al. (1981) de acuerdo a la tipología de formas societarias, implementando adicionalmente en una segunda fase de la investigación un estudio para evaluar los principales factores determinantes del rendimiento de dichas entidades en el periodo objeto de estudio.

Palabras clave: eficiencia, desempeño, eficiencia por programas, economía social, análisis envolvente de datos (DEA).

Claves Econlit: C140, C200, D240, P130

\footnotetext{
${ }^{1}$ Departamento de Economía Financiera y Contabilidad. Universidad Politécnica de Cartagena. Dirección de correo electrónico: isidoro.guzman@upct.es

${ }^{2}$ Departamento de Economía Aplicada. Universidad de Oviedo. Dirección de correo electrónico: aygarces26@yahoo.com

${ }^{3}$ Departamento de Economía Aplicada. Universidad de Oviedo. Dirección de correo electrónico: cramos@uniovi.es.
}

REVESCO No 110 - Primer Cuatrimestre 2013 - ISSN: 1885-8031 - www.ucm.es/info/revesco

http://dx.doi.org/10.5209/rev_REVE.2013.v110.41445

Fecha de recepción: 01/06/2012

Fecha de aceptación: 16/10/2012 


\title{
EFFICIENCY ANALYSIS BY PROGRAMS IN THE FIELD OF SOCIAL ECONOMY: THE CASE OF ASTURIAS
}

\begin{abstract}
This paper evaluates the efficiency of the Social Economy sector in Asturias, by applying the nonparametric method of Data Envelopment Analysis (DEA) proposed by Charnes et al. (1978). For the elaboration of this study we used a sample of 397 Asturian cooperatives and labor societies for biennium 2004-2005. We calculate the measure of its performance by applying the DEA technique to a model of performance based on the account of operating income under output orientation, using the methodology of program efficiency proposed by Charnes et al. (1981). In a second stage of this research we evaluate the main factors determining the performance of these entities in the same period of analysis.
\end{abstract}

Keywords: efficiency, performance, program efficiency, social economy, data envelopment analysis (DEA).

\section{LA ECONOMÍA SOCIAL EN EL PRINCIPADO DE ASTURIAS: PRINCIPALESENTIDADES REPRESENTATIVAS}

El término "Economía Social" es un concepto ampliamente discutido, al que también se hace referencia con otras locuciones tales como Tercer Sector o Economía Solidaria, que genéricamente aglutina a aquellas empresas u organizaciones que, no estando encuadradas en los sectores público o privado, se identifican por determinados rasgos distintivos específicos como el de finalidad de servicio a sus miembros con carácter prioritario al lucro, la toma de decisiones democrática, la autonomía de gestión y la preeminencia de la persona sobre el capital ante posibles repartos de rentas ${ }^{4}$.

\footnotetext{
${ }^{4}$ La Conferencia Europea Permanente de Cooperativas, Mutualidades, Asociaciones y Fundaciones (CEPCMAF) emitió en junio de 2002 la Déclaration finale commune des organisations européennes de l'Économie Sociales, poniendo de manifiesto los valores que congregan las entidades pertenecientes a la Economía Social, que son los siguientes (Monzon, 2006):

- Primacía de la persona y del objeto social sobre el capital.

- Adhesión voluntaria y abierta.

- Control democrático por sus miembros.

- Conjunción de los intereses de los miembros usuarios y del interés general.

- Defensa y aplicación de los principios de solidaridad y responsabilidad.

- Autonomía de gestión e independencia respecto de los poderes públicos.

- Destino de la mayoría de los excedentes a la consecución de objetivos a favor del desarrollo sostenible, del interés de los servicios a los miembros y del interés general.
} 
Sin entrar a distinguir que tipo de entidades se circunscriben al Sector de la Economía Social (en adelante SES), se puede afirmar que el mismo se integra fundamentalmente por sociedades cooperativas, sociedades laborales, mutualidades y asociaciones, habiendo adquirido durante los últimos años una significativa importancia en el desarrollo económico de las regiones, debido en gran medida a las características propias de las entidades que lo componen, que han venido influyendo de forma ostensible en aspectos tales como la capacidad de generación de empleo y el aumento del bienestar social.

La evolución del SES ha estado unida al creciente interés de investigadores, gobiernos y organismos supranacionales por analizar su importancia en las regiones e implementar políticas encaminadas a reforzar dicho sector, perspectiva que también ha sido compartida desde la Unión Europea. Sin embargo, paradójicamente, se dispone de escasa información del SES, sobre todo si se compara con los sectores público y privado, siendo hasta la fecha difícil disponer de informes periódicos que permitan conocer en detalle sus principales agregados macroeconómicos y su grado de desarrollo a nivel nacional, dificultad que se acrecienta si el objetivo es realizar análisis regionales, pues tal como están construidas las Cuentas Nacionales y Regionales, las correspondientes a este sector se encuentran difuminadas en los distintos sectores de la economía, y de ahí la dificultad para su medición.

Como en otras regiones españolas, el SES en Asturias muestra una relevante representación, en la cual las sociedades cooperativas y sociedades laborales pueden ser consideradas como las principales representantes del mismo. En concreto, según datos publicados por la Dirección General de Fomento de la Economía Social y del Fondo Social Europeo, en el ejercicio 2005 existían 828 empresas enmarcadas dentro de las distintas ramas de actividad económica, aportando un total de 3.599 empleos directos, aunque como anteriormente se ha mencionado, extrañamente el sector sigue siendo un gran desconocido en cuanto a sus principales magnitudes.

En el contexto descrito, el presente artículo se evalúa el rendimiento de las sociedades cooperativas y sociedades laborales radicadas en Asturias a partir de la metodología no paramétrica del Análisis Envolvente de Datos (Data Envelopment Analysis, DEA) propuesta en el trabajo seminal de Charnes et al. (1978).

El análisis de eficiencia descrito permitirá identificar, a partir de un conjunto de variables, aquellas entidades del SES con mejores prácticas de desempeño, facilitando su 
comparación por tipología empresarial (sociedades cooperativas vs. sociedades laborales) de acuerdo a la metodología DEA por programas, información que puede ser de especial relevancia para las administraciones públicas en el diseño de políticas que permitan mejorar el desarrollo del SES e incluso de la región, dado su especial alcance a nivel socioeconómico. Asimismo, esta información puede ser significativa para el propio SES, ya que al caracterizar las distintas entidades en términos de eficiencia, proporciona información relevante para la toma de decisiones encaminadas al mejor aprovechamiento de los recursos disponibles, principalmente para aquellas entidades menos eficientes.

Para la elaboración del estudio se utilizó la información de las cuentas anuales de una muestra de 397 sociedades cooperativas y sociedades laborales asturianas para el bienio 20042005, calculando la medida de su desempeño a partir de un modelo de rendimiento basado en la cuenta de resultados de explotación bajo orientación output, realizando un tratamiento por programas (Charnes et al. 1981) mediante el análisis de fronteras separadas para determinar en primer lugar la eficiencia de gestión (managerial efficiency) de acuerdo a la tipología de formas societarias integrantes de la muestra manejada, y posteriormente la eficiencia por programas o grupos (programmatic efficiency), con el fin de conocer el modelo societario que mejor rendimiento alcanza, complementándose el estudio en una segunda etapa con un análisis econométrico que evalúa los principales factores determinantes del rendimiento de dichas entidades en el periodo objeto de estudio.

El resto del trabajo se estructura como sigue: la sección segunda describe el panorama actual del sector de la economía social en Asturias, tanto de las empresas que lo componen como del nivel de empleo generado. La sección tercera introduce el concepto de eficiencia y las propuestas metodológicas para su cálculo, incluyendo los materiales utilizados y el diseño del modelo de rendimiento implementado. La sección cuarta contiene la discusión de los resultados empíricos obtenidos, y finalmente, en la sección quinta se recogen las conclusiones más relevantes de la investigación realizada.

\section{CARACTERIZACIÓN DE LAS SOCIEDADES COOPERATIVAS Y LABORALES ASTURIANAS}

La economía social asturiana está compuesta principalmente por sociedades laborales y cooperativas, pudiéndose apreciar (Cuadro 1) que de las 828 empresas existentes en 2005, algo más de dos tercios $(64,7 \%)$ corresponden a sociedades laborales, frente al tercio restante 
$(35,3 \%)$ perteneciente a cooperativas. Dichas entidades desarrollan su actividad económica principalmente en el sector servicios, al que pertenecen 528 empresas $(63,8 \%)$, seguido por sector industrial y de la construcción $(28,4 \%)$, quedando en último lugar el sector primario $(7,9 \%)$ con una cuota bastante reducida.

Profundizando en la composición del sector servicios, se aprecia que una cuarta parte de las empresas se dedica al comercio y reparación de vehículos, dejando entrever la importancia de esta actividad tanto en la economía social como en la regional. Asimismo, la rama de actividades inmobiliarias y de alquiler, y de servicios empresariales, también presenta un peso importante en la composición del sector terciario, alcanzando 15,9\% de las integradas en el mismo. En cuanto al sector industrial, cabe destacar principalmente la industria manufacturera, que representa el $14,7 \%$, en la que se incluyen actividades relevantes para la región como la metalurgia y la fabricación de productos metálicos, así como la industria de la alimentación, bebidas y tabaco.

Cuadro 1. Distribución de las Empresas dadas de alta en la Seguridad Social y Nivel de Empleo generado por el SES según Rama de Actividad (Año 2005)

\begin{tabular}{|c|c|c|c|c|c|c|c|c|c|c|c|c|}
\hline \multirow{2}{*}{ RAMA DE ACTIVIDAD } & \multicolumn{4}{|c|}{ Economía Social } & \multicolumn{4}{|c|}{ Cooperativas } & \multicolumn{4}{|c|}{ Sociedades Laborales } \\
\hline & $\begin{array}{c}\mathbf{N}^{\circ} \\
\text { Emp. }\end{array}$ & $\%$ & $\begin{array}{c}\mathbf{N}^{\circ} \\
\text { Trab. }\end{array}$ & $\%$ & $\begin{array}{c}\mathrm{N}^{\mathbf{o}} \\
\text { Emp. }\end{array}$ & $\%$ & $\begin{array}{c}\mathbf{N}^{\circ} \\
\text { Trab. }\end{array}$ & $\%$ & $\begin{array}{c}\mathbf{N}^{\mathbf{o}} \\
\text { Emp. }\end{array}$ & $\%$ & $\begin{array}{c}\mathbf{N}^{\mathbf{o}} \\
\text { Trab. }\end{array}$ & $\%$ \\
\hline $\begin{array}{l}\text { Agricultura, ganadería, caza, selvicultura y } \\
\text { pesca }\end{array}$ & 65 & 7,9 & 291 & 4,4 & 61 & 7,4 & 283 & 4,3 & 4 & $\mathbf{0 , 5}$ & 8 & 0,1 \\
\hline Industrias, energía y construcción & 235 & 28,4 & 1.956 & 29,5 & 81 & 9,8 & 702 & 10,6 & 154 & 18,6 & 1.254 & 18,9 \\
\hline Industrias extractivas & 2 & 0,2 & 22 & 0,3 & 0 & 0 & 0 & 0 & 2 & 0,2 & 22 & 0,3 \\
\hline Industria manufacturera & 122 & 14,7 & 1110 & 16,7 & 55 & 6,6 & 573 & 8,6 & 67 & 8,1 & 537 & 8,1 \\
\hline $\begin{array}{l}\text { Producción y distribución de energía eléctrica, } \\
\text { gas y agua }\end{array}$ & 12 & 1,4 & 3 & 0 & 12 & 1,4 & 3 & 0 & 0 & 0 & 0 & 0 \\
\hline
\end{tabular}




\begin{tabular}{|c|c|c|c|c|c|c|c|c|c|c|c|c|}
\hline Construcción & 99 & 12 & 821 & 12,4 & 14 & 1,7 & 126 & 1,9 & 85 & 10,3 & 695 & 10,5 \\
\hline Actividades de servicios & 528 & 63,8 & 4.381 & 66,1 & 150 & 18,1 & 2.614 & 39,4 & 378 & 45,7 & 1.767 & 26,7 \\
\hline $\begin{array}{l}\text { Comercio y reparación de todo tipo de } \\
\text { vehículos }\end{array}$ & 208 & 25,1 & 1480 & 22,3 & 64 & 7,7 & 1.017 & 15,3 & 144 & 17,4 & 463 & 7 \\
\hline Hostelería & 56 & 6,8 & 426 & 6,4 & 0 & 0 & 286 & 4,3 & 56 & 6,8 & 140 & 2,1 \\
\hline Transporte, almacenamiento y comunicaciones & 50 & 6 & 566 & 8,5 & 25 & 3 & 436 & 6,6 & 25 & 3 & 130 & 2 \\
\hline Intermediación financiera & 13 & 1,6 & 281 & 4,2 & 4 & 0,5 & 274 & 4,1 & 9 & 1,1 & 7 & 0,1 \\
\hline $\begin{array}{l}\text { Actividades inmobiliarias y de alquiler; } \\
\text { servicios empresariales }\end{array}$ & 132 & 15,9 & 806 & 12,2 & 31 & 3,7 & 17 & 0,3 & 101 & 12,2 & 789 & 11,9 \\
\hline Educación & 12 & 1,4 & 102 & 1,5 & 5 & 0,6 & 85 & 1,3 & 7 & 0,8 & 17 & 0,3 \\
\hline $\begin{array}{l}\text { Actividades sanitarias y veterinarias, servicio } \\
\text { social }\end{array}$ & 17 & 2,1 & 560 & 8,5 & 7 & 0,8 & 499 & 7,5 & 10 & 1,2 & 61 & 0,9 \\
\hline $\begin{array}{l}\text { Otras actividades sociales y de servicios } \\
\text { prestados a la comunidad; servicios personales }\end{array}$ & 40 & 4,8 & 160 & 2,4 & 14 & 1,7 & 0 & 0 & 26 & 3,1 & 160 & 2,4 \\
\hline TOTAL & 828 & 100 & 6.628 & 100 & 292 & 35,3 & 3.599 & 54,3 & 536 & 64,7 & 3.029 & 45,7 \\
\hline
\end{tabular}

Fuente: Dirección General de Fomento de la Economía Social y del Fondo Social Europeo. Ministerio de Trabajo e Inmigración. Registro de Cooperativas y Mercantil de Asturias

En cuanto al nivel de empleo de las sociedades cooperativas y laborales en Asturias, se situaba en 6.628 trabajadores para el año 2005 (Cuadro 1), de los que un 54,3\% lo aportaban las cooperativas y el $45,7 \%$ restante las sociedades laborales, destacando la importancia de las cooperativas como sociedades creadoras de empleo, pues pese a existir un menor porcentaje de este tipo de empresas, su nivel de empleo es el más elevado.

Por sectores y, en concordancia con el análisis realizado sobre la distribución del número de empresas, la cifra de trabajadores empleados en el sector servicios muestra un claro predominio para ambos tipos societarios, con porcentajes del 39,4\% y 26,7\% 
respectivamente para sociedades cooperativas y laborales. Respecto al resto de sectores, en cooperativas el segundo sector generador de empleo, a una gran distancia del sector servicios, es el industrial $(8,6 \%)$, estando por detrás el sector primario $(4,3 \%)$ y del de la construcción $(1,9 \%)$. Por su parte, para las sociedades laborales, después del sector servicios le siguen el de la construcción $(10,5 \%)$ y el industrial $(8,1 \%)$, quedando con una mínima participación el sector primario $(0,1 \%)$.

\section{METODOLOGÍA Y MATERIALES}

\subsection{Medida de la eficiencia}

La medida del rendimiento de cualquier unidad capaz de tomar decisiones económicas se presenta como una cuestión ciertamente compleja sobre la que es posible consultar un dilatado repertorio de literatura especializada sobre la materia (Bititici et al., 1997; Neely y Waggoner, 1998, entre otros). El concepto de eficiencia se utiliza para hacer patente el nivel de rendimiento que puede alcanzar una determinada unidad económica de decisión (decision making unit, DMU) respecto a su conjunto de posibilidades de producción de acuerdo a la tecnología existente, lo que se traduce en intentar conocer la posición adoptada en cuanto a si dicho nivel productivo se logra con el menor consumo de recursos o mediante la minimización del coste de producción, conceptos basados en los trabajos de Farrell (1957) que investigan la noción de eficiencia global. En este sentido, la eficiencia técnica o productiva evalúa la obtención de un determinado nivel de output según una predeterminada combinación de inputs, definiéndose la eficiencia en precio como la mejor combinación de inputs capaz de alcanzar el nivel estipulado de output con el menor coste, suponiendo conocidos los precios de los diferentes inputs empleados, calculándose la eficiencia global como el producto de la eficiencia técnica y en precio (Thanassoulis, 2001).

La metodología utilizada para la medida de la eficiencia se basa fundamentalmente en dos propuestas (Parkan, 2002): los modelos paramétricos, que parten de la especificación de la forma de la función de producción, utilizando técnicas matemáticas para la estimación de sus parámetros de acuerdo a los datos ofrecidos por las DMUs evaluadas (Coelli et al., 1998), y los modelos no paramétricos, que consideran las propiedades que debe satisfacer el conjunto de posibilidades de producción, estimando una frontera eficiente o de "buenas prácticas" conformada por las DMUs eficientes, sin que en este caso sea necesario asumir a priori una forma funcional para la función de producción (Thanassoulis, 2001). 
Al comparar las propuestas metodológicas expuestas se advierte que la aproximación no paramétrica exhibe como principales ventajas frente a la paramétrica su grado de flexibilidad para adaptarse a entornos multiproducto y ausencia de precios, así como no requerir la utilización de formas funcionales explícitas, si bien presenta ciertos inconvenientes tales como, entre otros, su carácter determinístico, que identifica cualquier desviación a la frontera de eficiencia como un comportamiento ineficiente de la DMU evaluada; la imposibilidad de medir la calidad de los outputs obtenidos, que se supone similar para todas las unidades en estudio, y la sensibilidad de los resultados a la presencia de outliers (Schuschny, 2007).

\subsection{La técnica no paramétrica del Análisis Envolvente de Datos (DEA)}

Como principales representantes del SES en Asturias, el presente trabajo evalúa el rendimiento de las cooperativas y sociedades laborales mediante la aplicación de la metodología no paramétrica determinística del Análisis Envolvente de Datos (Data Envelopment Analysis, DEA), capaz determinar un ratio multidimensional que proporciona un ranking de puntuaciones de eficiencia, a partir de los datos de producción suministrados por la muestra objeto de estudio.

Siguiendo la propuesta metodológica de Charnes et al. (1978), la formulación matemática del DEA se realiza a través de un modelo de programación lineal bajo el supuesto de que todas las DMUs están operando en su escala óptima (Modelo CRS, constant returns to scale), lo que permite obtener puntuaciones de eficiencia técnica global $\left(\mathrm{ET}_{\mathrm{CRS}}\right)$ sin considerar deseconomías de escala. En este sentido, es posible asumir una posible doble orientación, según sea el objetivo previamente programado para la investigación: la orientación-input identifica la mayor reducción radial de todos los consumos de inputs para obtener un determinado nivel de output, mientras que la orientación-output determina la máxima expansión radial de productos a partir de un determinado consumo de inputs.

El Modelo CRS (Charnes et al., 1978) propone la formulación matemática del DEA en orientación output a partir de la siguiente expresión matemática:

$$
\operatorname{Max} \theta_{z}
$$

s.a.: 


$$
\begin{array}{ll}
\sum_{j=1}^{n} \lambda_{j} X_{i j}+\mathrm{S}_{\mathrm{iz}}^{-}=X_{i z} & \mathrm{i}=1, \ldots \ldots ., \mathrm{m} \\
\sum_{j=1}^{n} \lambda_{j} Y_{r j}-\mathrm{S}_{\mathrm{rz}}^{+}=\theta_{\mathrm{z}} Y_{r z} & \mathrm{r}=1, \ldots \ldots ., \mathrm{p} \\
\theta_{z} \geq 0 ; \lambda_{j} \geq 0 & \mathrm{j}=1, \ldots \ldots ., \mathrm{n}
\end{array}
$$

donde los vectores $X_{i j}$ y $Y_{r j}$ recogen las cantidades de input $i$ y de output $r$ consumida y producida respectivamente por la $\mathrm{DMU} j$, mientras que la variable $\lambda_{j}$ expresa el peso de dicha entidad en la unidad virtual de referencia que puede ser construida por combinación lineal del resto de DMUs integrantes de la muestra evaluada. Si dicha unidad virtual no puede ser conseguida, entonces la DMU z para la que se soluciona el problema se considerará eficiente.

Resolviendo la formulación recogida (1)-(4) para cada DMU, el escalar $\theta_{\mathrm{z}}$ representa la mayor expansión radial de los outputs producidos por la unidad evaluada (DMU $z$ ), variando su rango entre 1 e $\infty$, de forma que tomará valor unitario cuando la unidad sea eficiente y valores superiores a 1 para el caso de unidades ineficientes, por lo que su puntuación de eficiencia técnica $\delta_{z}$ con rango entre 0 y 1 vendrá dada por la inversa del valor del escalar $\theta_{\mathrm{z}}\left(\delta_{\mathrm{z}}=1 / \theta_{\mathrm{z}}\right)^{5}$. Las variables $\mathrm{S}_{\mathrm{iz}}^{-}$y $\mathrm{S}_{\mathrm{rz}}^{+}$o slacks representan el aumento/reducción de los inputs/outputs del modelo, al margen del aumento radial de los outputs determinado por el escalar del modelo de eficiencia $\theta_{\mathrm{z}}$.

Dado que el Modelo CRS considera la hipótesis de rendimientos a escala constantes, y al objeto de evitar las dificultades asociadas a la medición de la eficiencia técnica en unidades sesgadas por ineficiencias de escala, Banker et al. (1984) propusieron un modelo alternativo mediante el que es posible asumir la hipótesis de rendimientos a escala variables (Modelo VRS, variable returns to scale) añadiendo al modelo de CRS la restricción $\sum_{j=1}^{n} \lambda_{j}=1$, lo que permite calcular puntuaciones de eficiencia técnica pura $\left(\mathrm{ET}_{\mathrm{VRS}}\right.$ ) considerando la escala de operaciones de las empresas eficientes respecto de la DMU evaluada en cada caso. En este sentido, si comparamos el plan productivo de una determinada DMU sobre la base de las

\footnotetext{
${ }^{5}$ En el caso de haber asumido la orientación input del modelo, el escalar $\theta_{\mathrm{z}}$ corresponderá a la mayor reducción radial del consumo de inputs de la unidad evaluada, cuyo rango variará entre 0 y 1 , de forma que será calificada de eficiente aquella unidad que obtenga un índice igual a 1, e ineficiente si su valor fuese inferior.
} 
fronteras de eficiencia de los modelos VRS y CRS, se puede determinar la eficiencia de escala (ES) de acuerdo a la formulación matemática presentada en (5)-(6):

$$
\begin{aligned}
& \mathrm{ES}=\mathrm{ET}_{\mathrm{CRS}} / \mathrm{ET}_{\mathrm{VRS}} \\
& \mathrm{ET}_{\mathrm{CRS}}=\mathrm{ET}_{\mathrm{VRS}} \times \mathrm{ES}
\end{aligned}
$$

donde $\mathrm{ET}_{\mathrm{CRS}}$ y $\mathrm{ET}_{\mathrm{VRS}}$ representan respectivamente los índices de eficiencia técnica global y pura de la DMU evaluada. Si el valor de la variable SE es igual a 1, la DMU en cuestión se encuentra en su escala óptima de operaciones, aflorando ineficiencias de escala cuando el valor de dicha variable se sitúa en niveles inferiores $(\mathrm{SE}<1)$.

Finalmente cabe puntualizar que el poder de discriminación de la técnica DEA está en función del número de variables integradas en el modelo de eficiencia respecto del número total de DMUs evaluadas $n$, siendo necesario que este último parámetro sea cuando menos el triple del total de inputs/outputs considerandos (El-Maghary y Ladhelma, 1995).

\subsection{Medida de la eficiencia por programas}

El trabajo seminal de Charnes et al. (1981) plantea la medida de la eficiencia por programas o grupos, a cuyos efectos distingue los conceptos de eficiencia de gestión (managerial o intra-program efficiency) que evalúa el rendimiento de una determinada DMU con respecto a la frontera de buenas prácticas dentro de su grupo de pertenencia, y eficiencia por programas (programmatic o inter-program efficiency), capaz de identificar diferencias de rendimiento entre los programas o grupos existentes procedentes exclusivamente de la propia idiosincrasia o naturaleza en su actuación productiva y que, en nuestro estudio, se identifica por la naturaleza jurídico-económica del modelo societario de pertenencia: sociedad cooperativa vs sociedad laboral.

En este sentido, para la estimación de la eficiencia por programas se deben realizar las siguientes fases:

a) División de la muestra en grupos o programas, aplicando a cada uno de ellos separadamente la formulación mostrada en (1)-(4) para obtener los niveles de eficiencia de gestión (managerial o intra-program efficiency).

b) A partir de los resultados de la eficiencia de gestión obtenidos en la fase a), aquellas DMUs que se hayan mostrado ineficientes deberán proyectarse sobre sus respectivas 
fronteras de buenas prácticas para eliminar las ineficiencias de gestión detectadas como consecuencia de las decisiones adoptadas por la dirección, para lo cual se deberán corregir los datos de producción originales aplicando la formulación mostrada en (7)-(8):

$$
\begin{aligned}
& \mathrm{y}_{\mathrm{rz}}^{\prime}=\theta_{z} * y_{r z}+S_{r z}^{+} \\
& \mathrm{x}^{\prime}{ }_{\mathrm{iz}}=x_{i z}-S_{i z}^{-}
\end{aligned}
$$

donde $\left(\mathrm{y}_{\mathrm{rz}}, \mathrm{x}_{\mathrm{iz}}{ }^{\prime}\right)$ representan los valores en frontera de los outputs e inputs de la DMU $\mathrm{z}$ evaluada, $\left(\mathrm{y}_{\mathrm{rz}}, \mathrm{x}_{\mathrm{iz}}\right)$ los datos originales de su proceso productivo, mientras que el escalar $\theta_{\mathrm{z}}$ y los slacks $\mathrm{S}_{\mathrm{iz}}^{-}$y $\mathrm{S}_{\mathrm{rz}}^{+}$procederán de la resolución del modelo de programación línea propuesto en (1)-(4).

c) Tomando los datos de producción "corregidos" obtenidos en la fase b), exentos de ineficiencias de gestión, se calcularán nuevamente las puntuaciones de eficiencia utilizando la formulación contenida en (1)-(4), si bien en este caso mediante el trazado de una frontera única de eficiencia sobre la totalidad de la muestra analizada, lo que permite el cálculo de los niveles de eficiencia por programas facilitando de esta forma la comparación del rendimiento de las DMUs perteneciente a cada uno de los grupos (o programas) evaluados.

d) Finalmente, para evaluar si existen diferencias de rendimiento estadísticamente significativas entre los programas analizados, será necesario practicar los correspondientes test estadísticos adecuados a tal efecto.

\subsection{Revisión de la literatura sobre la medida de la eficiencia en el SES}

Durante la última década han sido diversos los estudios realizados sobre eficiencia en el SES, tanto a nivel metodológico como empírico. En lo relativo al marco conceptual, podemos referirnos a artículos como el de Evans y Guthrie (2006), en el que se realiza un análisis técnico sobre las relaciones entre la eficiencia y el valor de la empresa. A nivel empírico destaca el trabajo de Sáez y González (2004), donde se estudia la importancia de las cooperativas y sociedades laborales en España, teniendo en cuenta algunos indicadores de competitividad tales como productividad, costes salariales y costes laborales unitarios.

En general, dentro de la literatura sobre eficiencia en el SES utilizando el método no paramétrico del DEA, predominan principalmente los estudios relacionados con las 
cooperativas agrícolas y de crédito (Sáez et al., 2011). Por lo que se refiere a las primeras, se pueden enumerar los trabajos de Sueyoshi et al. (1998) y Sueyoshi (1999), ambos para las cooperativas agrícolas japonesas; Singh et al. (2001) para las cooperativas del sector lácteo en la India y Krasachat y Chinkul (2009), en este caso para este tipo de sociedades en Tailandia. En España destacan los trabajos de Damas y Romero (1997) para el sector de las almazaras cooperativas en la provincia de Jaén; Vidal et al. (2000) y Martínez y Martínez-Carrasco (2002) en las cooperativas de comercialización hortofrutícola de la Comunidad Valenciana y Almería respectivamente; Montegut, et al. (2002) para las cooperativas productoras de aceite de oliva, y finalmente Guzmán et al. (2006 y 2008), que realizan un estudio mucho más amplio para las cooperativas agrarias a nivel nacional.

En cuanto a los trabajos enfocados a las cooperativas de crédito, Lang y Welzel (1999) emplean la técnica DEA con datos de panel para estimar el incremento en la eficiencia debido a la fusión de las cajas rurales en Alemania, y Marco y Moya (1999) también estiman una frontera de costes estocástica para estudiar la llamada "ineficiencia x" y las economías de escala en este tipo de empresas a nivel nacional. Análogamente, Miranda y Morales (2001) analizan la eficiencia de un grupo de cajas rurales y cajas de ahorro en España a partir de la construcción de la función de producción, y más recientemente, Goddard y Wilson (2005) evalúan el desempeño de las cooperativas de crédito en los Estados Unidos mediante análisis econométrico; Desrochers y Fischer (2005) realizan un estudio comparado entre varios países sobre el nivel de integración de los sistemas de cooperativas financieras y su influencia en las medidas de rendimiento; Ward y McKillop (2005) evalúan los características que pueden contribuir al rendimiento de las cooperativas de crédito del Reino Unido a nivel nacional y regional, a través del análisis econométrico con datos de corte transversal, y por último, Kontolaimou y Tsesouras (2010) investigan el comportamiento productivo de las empresas de la banca cooperativa en comparación con sus contrapartes comerciales y de ahorro con un enfoque metafrontera.

Aunque en menor medida, también existe evidencia empírica sobre el enfoque metodológico propuesto por Charnes et al. (1981) respecto a la cuantificación de la eficiencia por programas, tal como es posible apreciar en los trabajos presentados por Ahn et al. (1988), Muñiz (2001), Beltrán et al. (2011), García Rubio et al. (2010, 2011) y Gómez et al. (2011). En el primero de ellos se compara la eficiencia de las dos ordenaciones educativas LGE y LOGSE y en los dos últimos se implementa esta metodología para el sector agrario. Para el 
caso particular del SES, tan sólo se observa el trabajo presentado en Sáez et al. (2011), donde se confrontan la eficiencia por programas de las entidades de la economía social (cooperativas y sociedades laborales) pertenecientes al sector de la construcción español.

\subsection{Materiales}

A partir de la información contable de los Balances y Estado de Pérdidas y Ganancias (EPYG) obtenida de los Registros Mercantil y de Cooperativas del Principado de Asturias, se seleccionó una muestra de 397 empresas para el bienio 2004-2005 con la distribución que se muestra en el Cuadro 2, de donde se desprende que la muestra seleccionada representa el $47,95 \%$ de la población, de las cuales el $12,92 \%$ corresponde a las cooperativas y el 35,02\% a las sociedades laborales, acorde con la distribución poblacional del sector.

Cuadro 2. Distribución de la muestra por tipo societario

\begin{tabular}{|l|c|c|c|}
\hline \multirow{2}{*}{\multicolumn{1}{|c|}{ Tipo de sociedad }} & \multicolumn{2}{|c|}{ Número de empresas } & \multirow{2}{*}{$\begin{array}{c}\text { \% de } \\
\text { participación }\end{array}$} \\
\cline { 2 - 3 } & Muestras & Asturias & \\
\hline Sociedades Cooperativas & 107 & 292 & 12,92 \\
\hline Sociedades Laborales & 290 & 536 & 35,02 \\
\hline Total empresas seleccionadas & $\mathbf{3 9 7}$ & $\mathbf{8 2 8}$ & $\mathbf{4 7 , 9 5}$ \\
\hline
\end{tabular}

Fuente: Elaboración propia

Dada la escasa información a nivel microeconómico del SES en las diversas fuentes estadísticas públicas y el elevado costo de obtener la información, se tomó como periodo de referencia el bienio 2004-2005, espacio temporal para el cual se construyeron las primeras cuentas satélites del SES asturiano (Hurtado et al.; 2012).

Para evaluar el rendimiento de las citadas sociedades se analizó el modelo de eficiencia que se recoge en el Cuadro 3, cuyo diseño se realizó desde un punto de vista económico mediante la elección de las principales variables que integran el resultado de 
explotación de una compañía (Guzmán y Arcas, 2008; Singh et al., 2001), considerando para ello la orientación output del modelo dado que cualquier empresa aspira a la maximización de sus ventas netas a partir del consumo de factores productivos identificados por sus costes de producción.

Cuadro 3. Variables del modelo de eficiencia DEA

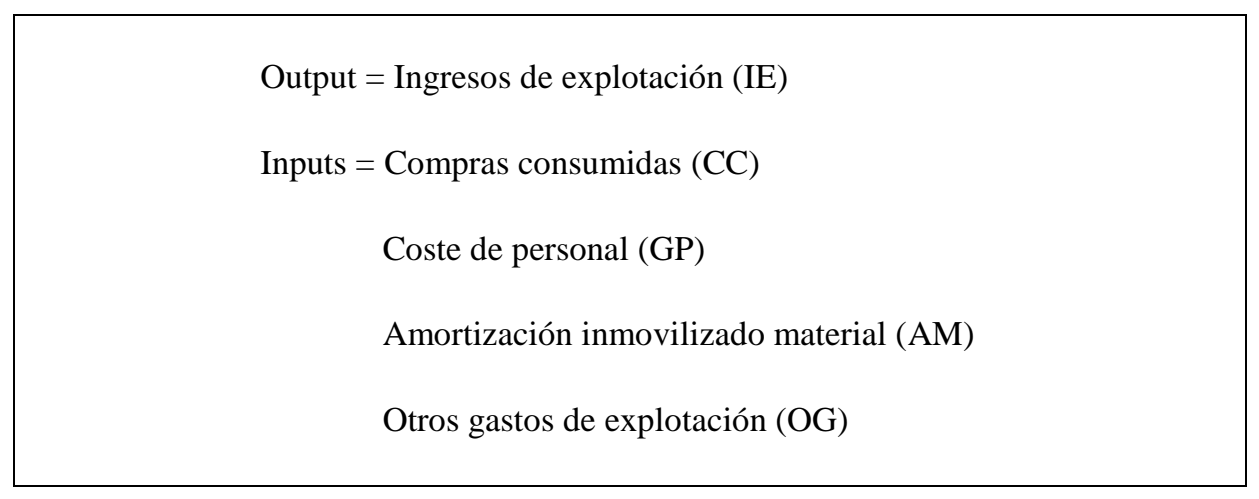

Fuente: Elaboración propia

El Cuadro 4 recoge los estadísticos descriptivos de las variables integradas en el modelo de eficiencia para los tipos societarios seleccionados.

Cuadro 4. Estadísticos descriptivos de las variables del modelo de eficiencia (miles euros)

Panel A: Sociedades Cooperativas $\left(N_{1}=107\right)$

\begin{tabular}{|c|c|c|c|c|c|c|}
\hline Periodo & Estadístico & IE & $\mathrm{CC}$ & GP & $\mathbf{A M}$ & OG \\
\hline \multirow{5}{*}{2004} & Mediana & 447,02 & 158,51 & 138,57 & 7,05 & 40,43 \\
\hline & Media & $3.922,65$ & $3.490,59$ & 267,08 & 29,06 & 145,59 \\
\hline & Des. típica & 20109,19 & 19402,80 & 558,95 & 66,82 & 360,64 \\
\hline & Máximo & $202.283,83$ & $195.385,45$ & $5.300,61$ & 489,54 & $2.918,53$ \\
\hline & Mínimo & 19,66 & 0,06 & 2,17 & 0,06 & 1,81 \\
\hline 2005 & Mediana & 476,29 & 160,87 & 133,71 & 8,36 & 43,81 \\
\hline
\end{tabular}

REVESCO No 110 - Primer Cuatrimestre 2013 - ISSN: 1885-8031 - www.ucm.es/info/revesco 
Cuadro 4. Estadísticos descriptivos de las variables del modelo de eficiencia (miles euros)

Panel A: Sociedades Cooperativas $\left(N_{1}=107\right)$

\begin{tabular}{|c|c|c|c|c|c|c|}
\hline Periodo & Estadístico & IE & $\mathbf{C C}$ & GP & $\mathbf{A M}$ & OG \\
\hline & Media & $4.072,95$ & $3.599,04$ & 279,44 & 33,90 & 161,75 \\
\hline & Des. típica & 20408,83 & 19642,26 & 582,24 & 83,35 & 409,47 \\
\hline & Máximo & $204.305,84$ & $196.888,26$ & $5.427,16$ & 686,32 & $3.271,62$ \\
\hline & Mínimo & 5,28 & 0,04 & 7,70 & 0,06 & 1,86 \\
\hline \multicolumn{7}{|c|}{ Panel B: Sociedades Laborales $\left(N_{2}=290\right)$} \\
\hline Periodo & Estadístico & IE & $\mathbf{C C}$ & GP & $\mathbf{A M}$ & OG \\
\hline \multirow{5}{*}{2004} & Mediana & 154,05 & 63,01 & 52,48 & 4,47 & 23,26 \\
\hline & Media & 337,85 & 169,83 & 111,51 & 9,49 & 44,99 \\
\hline & Des. típica & 613,45 & 303,42 & 291,60 & 20,63 & 74,84 \\
\hline & Máximo & $6.678,29$ & $2.119,08$ & $4.193,33$ & 248,56 & 746,28 \\
\hline & Mínimo & 6,27 & 0,12 & 2,70 & 0,13 & 2,62 \\
\hline \multirow{3}{*}{2005} & Mediana & 194,13 & 75,42 & 60,84 & 5,69 & 26,50 \\
\hline & Media & 390,46 & 192,25 & 129,72 & 10,87 & 52,44 \\
\hline & Des. típica & 647,64 & 319,79 & 324,07 & 26,24 & 86,67 \\
\hline
\end{tabular}


Cuadro 4. Estadísticos descriptivos de las variables del modelo de eficiencia (miles euros)

Panel A: Sociedades Cooperativas $\left(\mathrm{N}_{1}=107\right)$

\begin{tabular}{|ccccccc|}
\hline Periodo & Estadístico & IE & CC & GP & AM & OG \\
\hline \multirow{2}{*}{ Máximo } & $6.839,89$ & $2.160,92$ & $4.591,10$ & 368,37 & 723,47 \\
& & & & & 0,05 & 0,56 \\
\hline
\end{tabular}

Leyendas: IE = Ingresos de explotación; $\mathrm{CC}$ = compras consumidas; $\mathrm{GP}=$ Gastos de personal; $\mathrm{AM}=$ dotación amortización del ejercicio; $\mathrm{OG}=$ Otros gastos explotación.

Fuente: Elaboración propia a partir de la información de las cuentas anuales de las empresas de la muestra seleccionada.

\section{RESULTADOS EMPÍRICOS}

\subsection{Medida de la eficiencia de gestión (managerial o intra-program efficiency)}

De acuerdo a la metodología propuesta para el análisis de la eficiencia por programas, se resolvió el modelo de eficiencia (fase a) mediante la construcción de sendas fronteras separadas a partir de la tipología de formas societarias examinadas (sociedades cooperativas vs sociedades laborales).

Los resultados de la eficiencia intra-grupos se recogen en el Cuadro 5, donde se puede apreciar que el nivel medio de eficiencia técnica pura de las sociedades cooperativas es superior al de las sociedades laborales $(0,932$ vs 0,841$)$ para el bienio analizado, lo que de acuerdo a la orientación output del modelo de rendimiento evidencia que, en valores medios, las sociedades laborales deberían incrementar sus ingresos de explotación en un 18,90\% frente al 7,30\% de las sociedades cooperativas, aflorando una ineficiencia del $11,61 \%$ entre ambas formas societarias, que sin embargo, muestran un nivel similar de ineficiencia de escala en torno al $5 \%$. 
Cuadro 5. Puntuaciones de eficiencia de gestión (intra-efficiency)

\begin{tabular}{|c|c|c|c|c|c|c|}
\hline \multicolumn{7}{|c|}{ Panel A: Sociedades Cooperativas } \\
\hline & \multicolumn{2}{|c|}{ Modelo CRS } & \multicolumn{2}{|c|}{ Modelo VRS } & \multicolumn{2}{|c|}{ Eficiencia de Escala } \\
\hline Periodo & Media & Desv. típica & Media & Desv. típica & Media & Desv. típica \\
\hline 2004 & 0,896 & 0,116 & 0,942 & 0,106 & 0,951 & 0,049 \\
\hline 2005 & 0,872 & 0,161 & 0,922 & 0,125 & 0,948 & 0,120 \\
\hline Media anual & $\mathbf{0 , 8 8 4}$ & & 0,932 & & $\mathbf{0 , 9 5 0}$ & \\
\hline \multicolumn{7}{|c|}{ Panel B: Sociedades Laborales } \\
\hline & \multicolumn{2}{|c|}{ Modelo CRS } & \multicolumn{2}{|c|}{ Modelo VRS } & \multicolumn{2}{|c|}{ Eficiencia de Escala } \\
\hline Periodo & Media & Desv. típica & Media & Desv. típica & Media & Desv. típica \\
\hline 2004 & 0,793 & 0,167 & 0,833 & 0,163 & 0,954 & 0,086 \\
\hline 2005 & 0,794 & 0,126 & 0,848 & 0,118 & 0,940 & 0,091 \\
\hline Media anual & 0,794 & & 0,841 & & 0,947 & \\
\hline
\end{tabular}

Fuente: Elaboración propia

\subsection{Medida de la eficiencia por programas}

A partir de los resultados de la eficiencia intra-grupos (Cuadro 5) se eliminaron las ineficiencias de gestión (fase b), calculando posteriormente las puntuaciones de eficiencia por programas (fase c) que se presentan en el Cuadro 6, donde se observa que son las sociedades laborales las que, en valores medios anuales, presentan mejores niveles de rendimiento (modelo VRS: 0,974 vs 0,951; Modelo CRS: 0,929 vs 0,911). 
Cuadro 6. Puntuaciones Eficiencia por Programas (inter-efficiency)

\begin{tabular}{|c|c|c|c|c|c|c|}
\hline \multicolumn{7}{|c|}{ Panel A: Sociedades Cooperativas } \\
\hline & \multicolumn{2}{|c|}{ Modelo CRS } & \multicolumn{2}{|c|}{ Modelo VRS } & \multicolumn{2}{|c|}{ Eficiencia de Escala } \\
\hline Periodo & Media & Desv. típica & Media & Desv. típica & Media & Desv. típica \\
\hline 2004 & 0,912 & 0,065 & 0,947 & 0,051 & 0,963 & 0,043 \\
\hline 2005 & 0,910 & 0,125 & 0,956 & 0,115 & 0,951 & 0,059 \\
\hline Media anual & 0,911 & & 0,951 & & 0,957 & \\
\hline \multicolumn{7}{|c|}{ Panel B: Sociedades Laborales } \\
\hline & \multicolumn{2}{|c|}{ Modelo CRS } & \multicolumn{2}{|c|}{ Modelo VRS } & \multicolumn{2}{|c|}{ Eficiencia de Escala } \\
\hline Periodo & Media & Desv. típica & Media & Desv. típica & Media & Desv. típica \\
\hline 2004 & 0,930 & 0,095 & 0,971 & 0,055 & 0,958 & 0,081 \\
\hline 2005 & 0,927 & 0,092 & 0,978 & 0,030 & 0,948 & 0,090 \\
\hline Media anual & $\mathbf{0 , 9 2 9}$ & & 0,974 & & $\mathbf{0 , 9 5 3}$ & \\
\hline
\end{tabular}

Fuente: Elaboración propia

Atendiendo al test de diferencia de medias recogido en el Cuadro 7 (fase d), se observa que el rendimiento de ambos grupos de sociedades es estadísticamente significativo, por lo que se puede afirmar que el modelo empresarial representando por las sociedades laborales asturianas es recomendado frente al de sociedad cooperativa, al ser capaz de obtener un mayor rendimiento en dicha demarcación geográfica. 
Cuadro 7. Prueba $\mathrm{T}$ diferencia de medias por programas

\begin{tabular}{|c|c|c|c|c|c|c|}
\hline \multicolumn{7}{|c|}{ Prueba t para puntuaciones de Eficiencia Técnica Pura (Modelo VRS) } \\
\hline \multicolumn{3}{|c|}{ Sociedades Cooperativas } & \multicolumn{2}{|c|}{ Sociedades Laborales } & \multirow[b]{2}{*}{ Estad. T } & \multirow[b]{2}{*}{ P-value } \\
\hline Periodo & Media & Desv. típica & Media & Desv. típica & & \\
\hline 2004 & 0,947 & 0,051 & 0,971 & 0,055 & $-3,918$ & $0,000 * * *$ \\
\hline 2005 & 0,956 & 0,115 & 0,978 & 0,030 & $-3,009$ & $0,003 * * *$ \\
\hline M. agrupada & 0,951 & 0,089 & 0,974 & 0,044 & $-4,815$ & $0,000 * * *$ \\
\hline \multicolumn{7}{|c|}{ Prueba t para puntuaciones de Eficiencia Técnica Global (Modelo CRS) } \\
\hline & \multicolumn{2}{|c|}{ Sociedades Cooperativas } & \multicolumn{2}{|c|}{ Sociedades Laborales } & & \\
\hline Periodo & Media & Desv. típica & Media & Desv. típica & Estad. T & P-value \\
\hline 2004 & 0,912 & 0,065 & 0,930 & 0,095 & $-1,808$ & $0,071^{*}$ \\
\hline 2005 & 0,910 & 0,125 & 0,927 & 0,092 & $-1,457$ & 0,146 \\
\hline M. agrupada & 0,911 & 0,100 & 0,927 & 0,093 & $-2,287$ & $0,022 * *$ \\
\hline
\end{tabular}

Notas: *significativo al 10\%; ** significativo al 5\%; *** significativo al $1 \%$.

Fuente: Elaboración propia 


\section{FACTORES DETERMINANTES DE LA EFICIENCIA EN LAS ENTIDADES DE LA ECONOMÍA SOCIAL ASTURIANA: ANÁLISIS DEA EN DOS ETAPAS MEDIANTE REGRESIÓN LOGIT}

Para conocer los factores determinantes de los niveles de eficiencia alcanzados por las entidades representativas del SES en Asturias, se aplicó la metodología del análisis DEA en dos etapas, para lo cual se llevó a cabo un estudio econométrico tomando como variable dependiente las puntuaciones de eficiencia de gestión (modelo VRS) en función de diversas variables regresoras cuya información estuvo disponible.

La singularidad de la variable dependiente -puntuaciones de eficiencia- complica la elección del método econométrico a seleccionar, dado que dicha variable toma valor 1 cuando la DMU evaluada es eficiente y valores inferiores en el caso de situarse fuera de la frontera de "buenas prácticas" (Puig-Junoy, 2000). Así, desde el punto de vista estadístico, la distribución de los índices de eficiencia guarda cierta similitud con la de los modelos de regresión censurada $^{6}$, motivo por el cual un importante número de trabajos utilizan como método de estimación la regresión Tobit para examinar las variables exógenas que inciden en los citados niveles de rendimiento. Sin embargo, resulta difícil asumir en el caso que nos ocupa que la distribución de la variable dependiente se ajusta a la teoría de la regresión censurada, puesto que la acumulación de valores en el nivel de eficiencia más alto no es el resultado de un defecto en los datos, sino de la propia definición del problema, circunstancia a la que hay que añadir que en ocasiones no es posible asumir la condición de normalidad de dicha variable exigida por la regresión Tobit (Chilingerian,1995; González y Barber, 1996).

Por los motivos expuestos, para llevar a cabo el análisis econométrico se propuso como metodología más adecuada un modelo con variable dependiente dicotómica o regresión logit, técnica multivariante que permite estudiar la relación entre un conjunto de variables explicativas de naturaleza métrica o categórica respecto de una variable dependiente binaria, que sólo toma dos valores para especificar si el individuo analizado posee una determinada característica estudiada. Así, en el presente estudio la variable dependiente dicotómica (Y =

\footnotetext{
${ }^{6}$ En Estadística cabe distinguir entre muestras con datos truncados y censurados: en el primer caso -datos truncados- únicamente se consideran aquellas observaciones que satisfacen un cierto criterio, lo que en la realidad se traduce en trabajar con una submuestra de la variable original, mientras que en el segundo caso -datos censurados- se dispone de todas las observaciones originales, si bien algunas de ellas se alteran de acuerdo un criterio previamente concebido, por lo que la censura, a diferencia del truncamiento, se produce por un defecto de los datos de la muestra, ya que si no estuvieran censurados constituirían una muestra representativa de la población de interés no censurada (Sosa Escudero, 1999).
} 
$\{1,0\})$ tomará el valor 1 cuando la DMU evaluada sea eficiente y 0 en caso contrario, por lo que la probabilidad de que ocurra el suceso "ser eficiente" varía entre 0 y 1 a partir de la expresión mostrada en (9), mientras que la odd ratio $(\eta)$ o ventaja de producirse un suceso se muestra en la expresión (10), definiéndose la transformación logística como el logaritmo de la ventaja $(\eta)$ de la opción 1 (ser eficiente) frente a la opción 0 (ser ineficiente) según se indica en la expresión (11) (Jovel, 1995; Sánchez Vizcaíno, 2000):

$$
\begin{gathered}
p_{i}=\frac{e^{\alpha_{0}+\alpha_{1} x_{1}+\alpha_{2} x_{2}+\ldots . .+\alpha_{n} x_{n}}}{1+e^{\alpha_{0}+\alpha_{1} x_{1}+\alpha_{2} x_{2}+\ldots . .+\alpha_{n} x_{n}}} \\
\eta_{i}=\frac{p_{i}}{1-p_{i}}=e^{\alpha_{0}+\alpha_{1} x_{1}+\alpha_{2} x_{2}+\ldots . .+\alpha_{n} x_{n}} \\
\ln \eta_{i}=\ln \left[\frac{p_{i}}{1-p_{i}}\right]=\alpha_{0}+\alpha_{1} x_{1}+\ldots+\alpha_{n} x_{n}
\end{gathered}
$$

Un problema adicional es la posibilidad de aplicar el modelo de regresión logística con datos de panel, lo que permite combinar la información de diversos individuos en un momento concreto (corte transversal) durante varios periodos de tiempo (serie temporal) para conocer la heterogeneidad inobservable a través del efecto individual, que puede deberse a características particulares de las unidades observadas no recogidas en las variables explicativas de la regresión.

Por consiguiente, la primera cuestión que surge sobre la aplicación de la regresión con datos de panel es averiguar si sería más factible alternativamente realizar una regresión convencional sobre la muestra agrupada de las observaciones conocidas para todos los periodos disponibles (pooled sample), problema que puede resolverse mediante la aplicación del Test de Breusch-Pagan o Multiplicador de Lagrange, que considera como hipótesis nula la aplicación del modelo de regresión logit sobre la muestra agrupada, que si es rechazada (pvalue $<0,05)$ implica como mejor opción la elección el modelo de regresión con datos de panel (Breusch y Pagan, 1980) ${ }^{7}$.

\footnotetext{
${ }^{7}$ En el supuesto de asumir la existencia de heterogeneidad de acuerdo a los resultados del test de Breusch y Pagan, su tratamiento puede realizarse a través de la estimación de los modelos de efectos aleatorios y fijos, que
} 
Partiendo de los razonamientos metodológicos expuestos, para computar los factores determinantes de la efíciencia se diseñó un modelo de regresión logit (Cuadro 8) considerando como variable endógena las puntuaciones del modelo rendimiento a escala variables (modelo VRS) de las sociedades cooperativas y laborales analizadas en el periodo objeto de estudio (bienio 2004-2005), si bien, para evaluar la sensibilidad de los coeficientes de los regresores se reclasificó la variable dicotómica considerando tres puntos de corte $(1,00$; 0,98 y 0,95), aceptándose la significatividad estadística de las variables independientes bajo la premisa de obtener un nivel de significación del 5\% o inferior en los tres modelos evaluados para cada uno de los grupos de sociedades estudiadas.

Cuadro 8. Modelo de Regresión Logit

$$
\mathrm{DEA}_{\text {it }}=\rho_{0}+\rho_{1} \mathrm{TAM}_{\text {it }}+\rho_{2} \mathrm{ANT}_{\mathrm{it}}+\rho_{3} \mathrm{NHT}_{\text {it }}+\rho_{4} \mathrm{LIQ}_{\mathrm{it}}+\rho_{5} \mathrm{END}_{\mathrm{it}}+\rho_{6} \mathrm{AUT}_{\mathrm{it}}+\mathrm{u}_{\mathrm{it}}
$$

donde:

DEA $_{\mathrm{it}}$ : Variable dicotómica $(1,0)$ según puntuación de eficiencia de la DMU i en el periodo t.

$\mathrm{TAM}_{\mathrm{it}}$ : Total activo de la DMU i en el periodo $\mathrm{t}$.

$\mathrm{ANT}_{\mathrm{it}}$ : Número de años desde la creación de la DMU i en el periodo t.

$\mathrm{NHT}_{\mathrm{it}}$ : Número de habitantes de la población donde radica la DMU i en el periodo t.

LIQ $_{\mathrm{it}}$ : Ratio de liquidez de la DMU i en el periodo t.

$\mathrm{END}_{\mathrm{it}}$ : Ratio de endeudamiento de la DMU i en el periodo t.

$\mathrm{AUT}_{\mathrm{it}}:$ Ratio de capitalización de la DMU i en el periodo t.

Fuente: Elaboración propia

generan respectivamente estimaciones más eficientes y consistentes que la regresión logit convencional (Arellano y Honoré, 1999), aplicando el test de Hausman (1978) para determinar la selección del modelo a evaluar (Wooldridge, 2002; Hsiao, 2003). 
Dado que se dispone de un panel de datos balanceado para el bienio 2004-2005, se aplicó el test de Breusch-Pagan, cuyos resultados (Cuadro 9) justifican la elección del modelo de regresión sobre la muestra agrupada frente al de regresión anidada en todos los modelos propuestos.

Cuadro 9. Test de Breusch - Pagan

\begin{tabular}{|c|c|c|c|}
\hline \multicolumn{4}{|c|}{ Panel A: Sociedades Cooperativas } \\
\hline & Modelo & Chi (2) & P-value \\
\hline 1,00 & Modelo 1 - Punto de corte: & 0,98 & 0,321 \\
\hline 0,98 & Modelo 2 - Punto de corte: & 0,95 & 0,330 \\
\hline 0,95 & Modelo 3 - Punto de corte: & 1,00 & 0,317 \\
\hline \multicolumn{4}{|c|}{ Panel B: Sociedades Laborales } \\
\hline & Modelo & Chi (2) & P-value \\
\hline 1,00 & Modelo 4 - Punto de corte: & 0,84 & 0,360 \\
\hline 0,98 & Modelo 5 - Punto de corte: & 1,00 & 0,318 \\
\hline 0,95 & Modelo 6 - Punto de corte: & 0,99 & 0,320 \\
\hline
\end{tabular}

Fuente: Elaboración propia 
Los Cuadros 10 y 11 presentan los resultados del modelo de regresión propuesto aplicado sobre la muestra agrupada para sociedades cooperativas (modelos 1, 2 y 3) y sociedades laborales (modelos 4,5 y 6 ) respectivamente, donde es posible constatar un alto grado de consistencia para los tres puntos de corte seleccionados, dado que en todos los casos las variables alcanzan o no significatividad estadística de forma uniforme en todos ellos.

Por tipos de sociedades se observa que de las seis variables explicativas analizadas (Cuadro 8), es la referida al tamaño (TAM) la que presenta un coeficiente positivo y significativo en todos los modelos al nivel del $1 \%$-excepto en el modelo 1 que es significativa al 5\%-, si bien su contribución a que la empresa sea calificada como eficiente es escasa de acuerdo al valor de su odd ratio (Cuadro 10). Además, para el caso exclusivo de la tipología de sociedades laborales, también resultan significativas al nivel del $1 \%$ la variables antigüedad (ANT) y ratio de autonomía (AUT), en el primer caso con signo negativo y el segundo con signo positivo, lo que implica que la probabilidad de que la compañía sea calificada como eficiente disminuye al aumentar el número de años desde su creación (Cuadro 11: odd ratio $<1$ : horquilla 0,885 a 0,917$)$, mientras que crece cuando se financia con fondos propios (Cuadro 11: odd ratio > 1: 1,177 - 1,189).

Las restantes variables regresoras relativas a dimensión demográfica (NHT), liquidez (LIQ) y endeudamiento (END) no resultaron estadísticamente significativas en ninguno de los modelos propuestos, por lo que en los términos descritos no se consideró concluyente su efecto respecto a la probabilidad de calificar una empresa como eficiente. 
Cuadro 10. Resultados Regresión Logit para Sociedades Cooperativas (muestra agrupada)

Variable dependiente: Puntuación DEA (modelo VRS) - Rango de datos: 2004 - 2005

\begin{tabular}{|c|c|c|c|c|c|c|c|c|c|c|c|c|c|c|c|}
\hline \multirow{2}{*}{ Modelo } & Interc & \multicolumn{2}{|c|}{ TAM } & \multicolumn{2}{|c|}{ ANT } & \multicolumn{2}{|c|}{ NHT } & \multicolumn{2}{|c|}{ LIQ } & \multicolumn{2}{|c|}{ END } & \multicolumn{2}{|c|}{ AUT } & & \\
\hline & $\begin{array}{c}\text { Coefic. } \\
(\mathbf{z})\end{array}$ & $\begin{array}{l}\text { Coefic. } \\
\text { (z) }\end{array}$ & $\begin{array}{l}\text { Odds } \\
\text { ratios }\end{array}$ & $\begin{array}{c}\text { Coefic. } \\
(\mathbf{z})\end{array}$ & $\begin{array}{l}\text { Odds } \\
\text { ratios }\end{array}$ & $\begin{array}{c}\text { Coefic. } \\
\text { (z) }\end{array}$ & $\begin{array}{l}\text { Odds } \\
\text { ratios }\end{array}$ & $\begin{array}{c}\text { Coefic. } \\
\text { (z) }\end{array}$ & $\begin{array}{l}\text { Odds } \\
\text { ratios }\end{array}$ & $\begin{array}{l}\text { Coefic. } \\
(\mathbf{z})\end{array}$ & $\begin{array}{l}\text { Odds } \\
\text { ratios }\end{array}$ & $\begin{array}{c}\text { Coefic. } \\
(\mathbf{z})\end{array}$ & $\begin{array}{l}\text { Odds } \\
\text { ratios }\end{array}$ & Chi2 & PseudoR $^{2}$ \\
\hline $\begin{array}{l}\text { Modelo } 1 \\
\text { P.c.: } 1,00\end{array}$ & $\begin{array}{l}-0,836 \\
(-1,53)\end{array}$ & $\begin{array}{c}1,03 \mathrm{E}-07^{* *} \\
(2,10)\end{array}$ & 1,000 & $\begin{array}{l}-0,008 \\
(-0,59)\end{array}$ & 0,992 & $\begin{array}{c}-3,84 \mathrm{E}-07 \\
(-0,23)\end{array}$ & 0,999 & $\begin{array}{l}0,117 \\
(0,87)\end{array}$ & 1,124 & $\begin{array}{l}-0,720 \\
(-1,04)\end{array}$ & 0,487 & $\begin{array}{l}-0,085 \\
(-0,80)\end{array}$ & 0,918 & $10,82^{*}$ & 0,048 \\
\hline
\end{tabular}




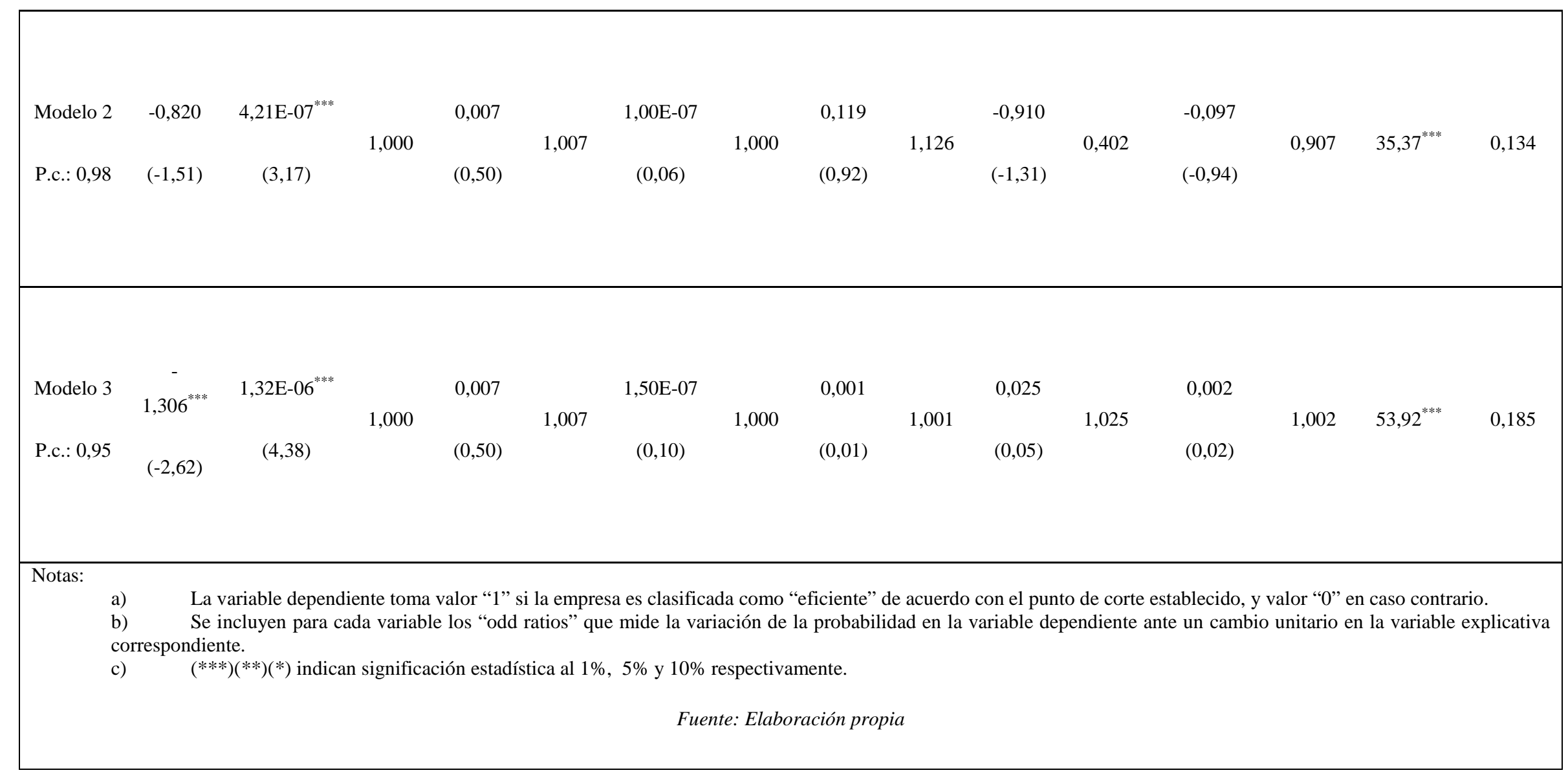

REVESCO No 110 - Primer Cuatrimestre 2013 - ISSN: 1885-8031 - www.ucm.es/info/revesco 
Cuadro 11. Resultados Regresión Logit para Sociedades Laborales (muestra agrupada)

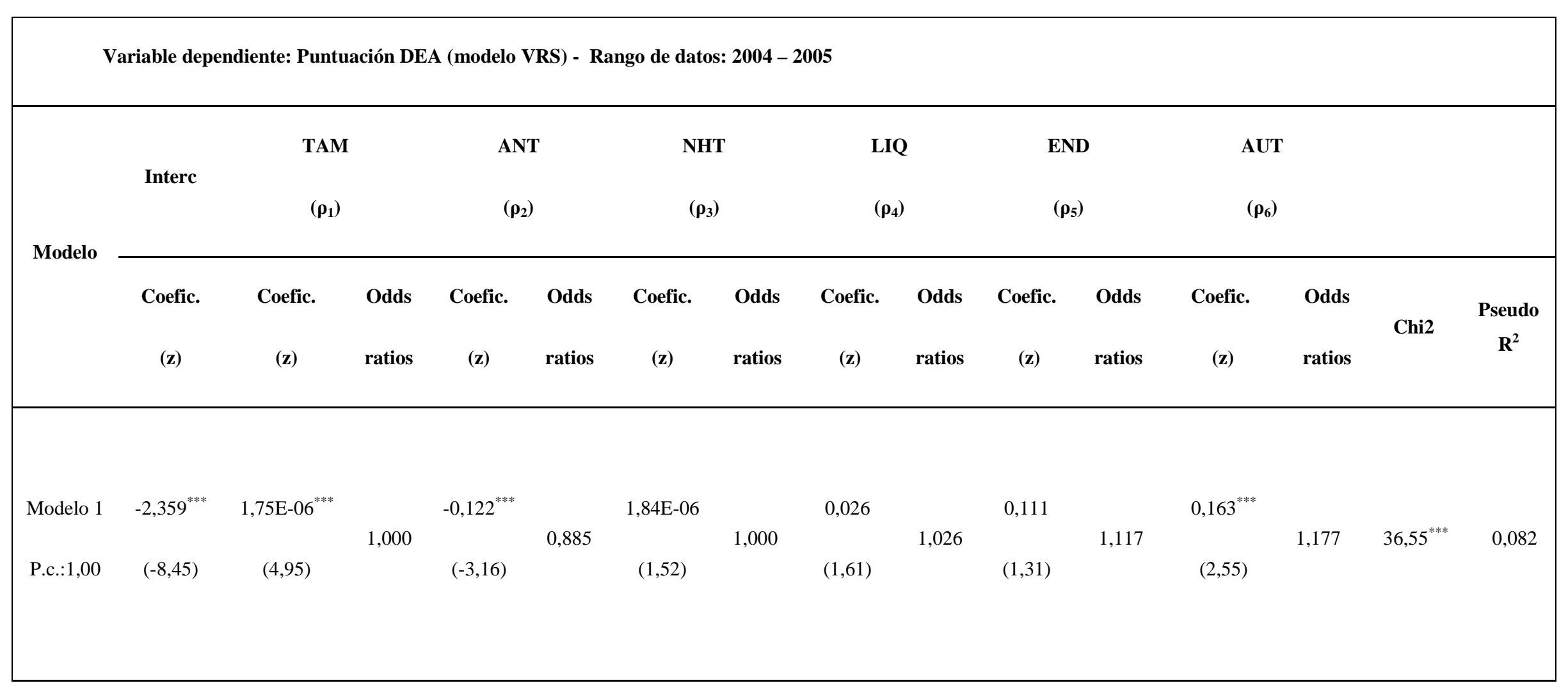




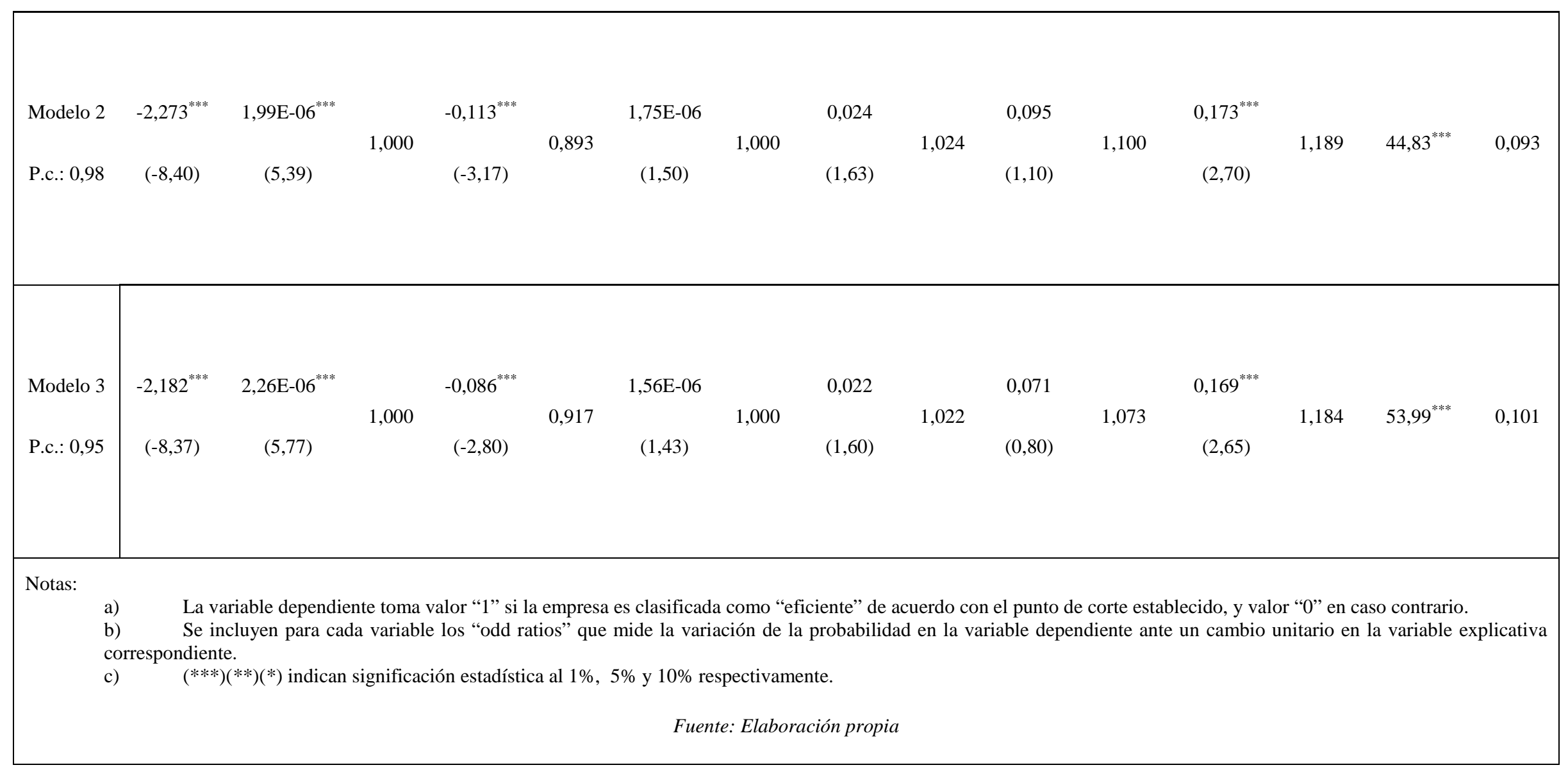




\section{CONCLUSIONES}

El presente estudio analiza el nivel de eficiencia del Sector de la Economía Social en Asturias representado por las cooperativas y sociedades laborales para el bienio do 20042005, a partir del trazado de fronteras eficientes mediante la técnica no paramétrica DEA, para lo cual se aplicó un modelo de rendimiento basado en información contable procedente de las cuentas de resultados de dichas compañías.

Aplicando la metodología del análisis de eficiencia por programas (grupos) propuesta por Charner et al. (1981), nuestros resultados evidencian, en valores medios, que los niveles de eficiencia de gestión (managerial o intra-efficiency) bajo la hipótesis de rendimientos a escala variables (modelo VRS) de las sociedades cooperativas son superiores a los presentados por las sociedades laborales $(0,932$ vs 0,841$)$, si bien, una vez eliminadas las ineficiencias de gestión, la eficiencia por programas (programmatic o inter-efficiency) presenta como modelo empresarial recomendado al de las sociedades laborales frente al de las sociedades cooperativas, que exhiben un rendimiento superior $(0,974$ vs 0,951$)$, existiendo diferencias estadísticamente significativas entre ambos modelos societarios de acuerdo a los test estadísticos de diferencias de medias practicados.

En cuanto a los factores determinantes de la eficiencia de gestión, los resultados del análisis DEA en dos etapas permiten concluir que la variable tamaño resultó estadísticamente significativa y con signo positivo para ambos tipos de sociedades. Adicionalmente, y únicamente para la tipología de sociedades laborales, también resultaron estadísticamente significativas las variables antigüedad y ratio de autonomía financiera, en el primer caso con signo negativo y en el segundo positivo, lo que implica que la antigüedad de dichas empresas disminuye su probabilidad de situarse en la frontera de buenas prácticas, mientras que una estructura financiera basada en las aportaciones de los socios y la autofinanciación de la compañía aumenta tal perspectiva. Sin embargo, los regresores relacionados con la demografía de la zona geográfica en que radican las empresas, así como los ratios de liquidez y endeudamiento, no fueron estadísticamente significativos en ninguno de los modelos propuestos, por lo que no es posible llegar a conclusiones sobre los datos ofrecidos por tales variables explicativas. 
No obstante, los resultados anteriormente comentados deben ser interpretados con precaución, pues debido al elevado costo y dificultad para obtener la información con fecha posterior al 2005, no pudieron ser evaluados un mayor número de ejercicios, análisis que se incluye dentro de las futuras líneas de investigación a plantear.

\section{BIBLIGRAFÍA}

AHN, T., CHARNES, A. y COOPER, W.W. (1988), "Some statistical and DEA evaluations of relative efficiencies of public and private institutions of higher education", SocioEconomic Planning Science, 22 (6), p. 259-269.

ARELlANO, M. y HONORÉ, B. (1999), "Panel data models: Some recent evidence", Working Paper, Princeton University, http://www.princeton.edu/ honore/papers.

BANKER, R.D., CHARNES, A. y COOPER, W.W. (1984), "Some models for estimating technical and scale inefficiencies in Data Envelopment Analysis", Management Science, 30, p. 1078-1092.

BELTRÁN, M., GÓMEZ, J.A. y PICAZO, A.J. (2011), “Are production technologies associated to agri-environmental programs more eco-efficient? A case study for rain-fed agriculture", Working paper WPAE-1115, Universidad de Valencia, Departamento de Estructura Económica.

BITITICI, U., CARRIE, A.S. y MCDEVITT, L. (1997), "Integrated performance measurement systems: a development guide", International Journal of Operations \& Production Management, 17, 5, p. 522-534.

BREUSCH, T. y PAGAN, A. (1980), “The Lagrange multiplier and its applications to model specification in econometrics", Review of Economics Studies. 47, p. 239-253.

CHARNES, A., COOPER, W.W. y RHODES, E. (1978), "Measuring the efficiency of decision making units", European Journal of Operational Research, 2, p. 429-444.

CHARNES, A., COOPER, W.W. y RHODES, E. (1981), "Evaluating program and managerial efficiency: an application of data envelopment analysis to program follow through", Management Science, 27 (6), p. 668-697.

CHILINGERIAN, J.A. (1995), "Evaluating physician efficiency in hospitals: A multivariate analysis of best practices", European Journal of Operational Research, 80, 3, p. 548-574.

COELLI, T., PRASADA RAO, D.S. y BATTESE, G.E. (1998), An Introduction to Efficiency and Productivity Analysis, Kluwer Academic Publishers, Massachussetts, USA. 
DAMAS, E. y ROMERO, C. (1997), “Análisis no paramétrico de la eficiencia relativa de las almazaras cooperativas en la provincia de Jaén durante el período 1975-1993”, Revista de Economía Agraria, 180, p. 279-304.

DESROCHERS, M. y FISCHER, K.P. (2005), "The power of networks: Integration and financial cooperative performance", Annals of Public and Cooperative Economics, 76, 3, p. 307-354.

EL-MAGHARY, S. y LADHELMA, R. (1995), "Data Envelopment Analysis: visualizing the results”, European Journal of Operational Research, 85, p. 700-710.

EVANS, L. y GUTHRIE, G. (2006), “A dynamic theory of cooperatives: The link between efficiency and valuation", Journal of Institutional and Theoretical Economics, 162, 2, p. 364-383.

FARRELL, M.J. (1957), "The measurement of productive efficiency", Journal of Royal Statistical Society Series, 120, p. 253-281.

GARCÍA RUBIO, M.A., GONZÁlEZ GÓMEZ, F. y GUARDIOLA, J. (2010), "Performance and ownership in the governance of urban water", Municipal Engineer, 163, p. $51-58$.

GARCÍA RUBIO, M.A., PICAZO TADEO, A. y GONZÁLEZ GÓMEZ, F. (2011), "Does a red shirt improve sporting performance? Evidence from Spanish football", Applied Economics Letters, i-first, p. 1-4.

GODDARD, J.A. y WILSON, J.O.S. (2005), "US Credit Unions: An empirical investigation of size, age and growth", Annals of Public and Cooperative Economics, 76, 3, p. 375-406.

GÓMEZ, J.A., PICAZO, A.J. y REIG, E. (2011), "Eco-efficiency assessment of olive farms in Andalusia", Working Paper WPAE-1105, Departamento de Estructura Económica, Universidad de Valencia, Valencia.

GONZÁLEZ, B. y BARBER, P. (1996): "Changes in the efficiency of Spanish Public Hospitals after the introduction of program-contracts", Investigaciones Económicas, vol. XX (3), septiembre, p. 377-402.

GUZMÁN, I., ARCAS, N. y GARCÍA, D. (2006), "La eficiencia técnica como medida del rendimiento de las cooperativas agrarias", Revista de Economía Publica, Social y Cooperativa, Ciriec-España, 55, p. 289-311.

GUZMÁN, I. y ARCAS, N. (2008), “The usefulness of accounting information in the measurement of technical efficiency in agricultural cooperatives", Annals of Public and Cooperative Economics, 79, 1, p. 107-131. 
HAUSMAN, J.A. (1978), "Specification test in econometrics", Econometrica, 46, p. 12511271.

HURTADO, A., RAMOS, C. y FERNÁNDEZ, E. (2012), "Elaboración de las Cuentas Satélite de las entidades de la Economía Social. Un estudio de caso para la Economía Asturiana en el año 2005" Ciriec-España, № 74, p. 5-37.

HSIAO, C. (2003), Analysis of Panel Data, Cambridge University Press, New York (USA).

JOVEL, A.J. (1995): Análisis de regresión logística, Centro de Investigaciones Sociológicas, Madrid.

KONTOLAIMOU, A. y TSEKOURAS, K. (2010), “Are cooperatives the weakest link in European banking? A non-parametric meta-frontier approach", Journal of Banking and Finance, 34, 8, p. 1946-1957.

KRASACHAT, W. y CHINKUL, K. (2009), "Performance measurement of agricultural cooperatives in Thailand: An accounting-based data envelopment analysis", in Lee, A. and Hashuti, A. (Eds.), Productivity, efficiency and economic growth in Asia-Pacific region, Physica-Verlag.

LANG, G. y WELZEL, P. (1999), "Mergers among German cooperative banks: A panelbased stochastic frontier analysis", Small Business Economics, 13, p. 273-286.

MARCO, M.A. y MOYA, I. (1999), "Inefficiency in the Spanish cooperative banking sector", Annals of Public and Cooperative Economics, 70, 4, p. 621-637.

MARTÍNEZ, J.M. y MARTÍNEZ-CARRASCO, F. (2002), "Las empresas de comercialización hortícola de Almería: análisis no paramétrico de eficiencia técnica", Revista Española de Estudios Agrosociales y Pesqueros, 197, p. 105-128.

MIRANDA, A. y MORALES, A.C. (2001), “Les Caisses Rurales face a L'Union Monetaire. Une etude d'efficacite compare", Annals of Public and Cooperative Economics, 72, 1, p. 135- 154

MONTEGUT, Y., SABATÉ, P. y CLOP, M.M. (2002), “Análisis de la eficiencia del asociacionismo cooperativo en la 'D.O. Garrigues' Lleida', Investigación Agraria: Producción Vegetal, 17 (3), p. 441-456.

MONZÓN CAMPOS, J.L. (2006), "Economía Social y conceptos afines: fronteras borrosas y ambigüedades conceptuales del Tercer Sector”, Revista de Economía Social, Pública y Cooperativa Ciriec-España, no 56, p. 9-24.

MINISTERIO DE TRABAJO Y ASUNTOS SOCIALES (2005), Estadísticas de Economía Social, Dirección General de Fomento de la Economía Social y del Fondo Social Europeo. 
MUÑIZ PÉREZ, M. (2001): “Son realmente menos eficientes los centros LOGSE?”, Hacienda Pública Española, vol. 157(2): p. 169-196.

NEELY, A. y WAGGONER, D. (1998), Performance Measurement: Theory and Practice, University of Cambridge Press, Cambridge, 1998.

PARKAN, C. (2002), "Measuring the operational performance of a public transit company", International Journal of Operations \& Production Management, 22, 6, p. 693-720.

PASTOR, J.M. (1995), "Eficiencia, cambio productivo y cambio técnico en los bancos y cajas de ahorro españolas: un análisis de la frontera no paramétrico", Revista Española de Economía, 12, p. 35-73.

PUIG-JUNOY, J. (2000), "Eficiencia en la atención primaria de salud: una revisión crítica de las medidas de frontera", Revista Española de Salud Pública, 74, p. 483-495.

SÁEZ, F.J. y GONZÁLEZ, F. (2004), "Productividad y costes salariales en el subsector de cooperativas y sociedades laborales", Economistas, 102, p. 50-55.

SÁEZ, F.J., PICAZO, A.J. y LLORCA, C. (2011), "Do Labour Societies Perform Differently to Cooperatives? Evidence from the Spanish Building Industry", Working Paper WPAE2011-09, Departamento de Estructura Económica, Universidad de Valencia, Valencia.

SÁNCHEZ VICAÍNO, G. (2000), "Regresión Logística” en Luque Martínez, T. Técnicas de análisis de datos en investigación de mercados, Editorial Pirámide, Madrid.

SCHUSCHNY, A.R. (2007), El método DEA y su aplicación al estudio del sector energético y las emisiones de CO2 en América Latina y el Caribe, Serie Estudios Estadísticos y Prospectivos, División de Estadística y Prospecciones Económicas, Comisión Económica para Ameria Latina y el Caribe (CEPAL), Naciones Unidas, Santiago de Chile.

SINGH, S., COELLI, T. y FLEMING, E. (2001), "Performance of dairy plants in the cooperative and private sectors in India", Annals of Public and Cooperative Economics, 72 (4), p. 453-479.

SOSA ESCUDERO, W. (1999), Tópicos de Econometría Aplicada, trabajo Docente Nro. 2, Universidad de Mar del Plata, Argentina.

SUEYOSHI, T. (1999), "DEA non-parametric ranking test and index measurement: slack adjusted-DEA and an application to Japanese agriculture cooperatives", International Journal of Management Science, 27:3, p. 315-326.

SUEYOSHI, T., HASEBE, T., ITO, F., SAKAI, J. y OZAWA, W. (1998), "DEA bilateral performance comparison: An application to Japan agricultural co-operatives (Nokyo)", International Journal of Management Science, 26, 2, p. 233-248. 
THANASSOULIS, E. (2001), Introduction to the Theory and Application of Data Envelopment Analysis, Ed. Kluwer Academic Publishers, Netherlands.

VIDAL, F., SEGURA, B. y DEL CAMPO, F.J. (2000), "Eficiencia de las cooperativas de comercialización hortofrutícola de la Comunidad Valenciana”, Revista de Estudios Agrosociales y Pesqueros, 188, p. 205-224.

WARD, A.M. y MCKILLOP, D.G. (2005), "An investigation into the link between UK Credit Union characteristics, location and their success", Annals of Public and Cooperative Economics, 76, 3, p. 461-489.

WOOLDRIDGE, J.M. (2002), The econometric analysis of cross section and panel data, The Massachusetts Institute of Technology Press, Cambridge. 